\title{
A Dynamical Perspective on the Generality Problem
}

\author{
Andreas Stephens $^{1}$ (D) - Trond A. Tjøstheim ${ }^{1} \cdot$ Maximilian K. Roszko $^{1}$ • \\ Erik J. Olsson ${ }^{1}$
}

Received: 21 July 2020 / Accepted: 11 December 2020 / Published online: 12 January 2021

(C) The Author(s) 2021

\begin{abstract}
The generality problem is commonly considered to be a critical difficulty for reliabilism. In this paper, we present a dynamical perspective on the problem in the spirit of naturalized epistemology. According to this outlook, it is worth investigating how token belief-forming processes instantiate specific types in the biological agent's cognitive architecture (including other relevant embodied features) and background experience, consisting in the process of attractorguided neural activation. While our discussion of the generality problem assigns "scientific types" to token processes, it represents a unified account in the sense that it incorporates contextual and common sense features emphasized by other authors.
\end{abstract}

Keywords Dynamical systems · Naturalistic epistemology $\cdot$ Reliabilism · The generality problem

\section{Introduction}

According to process reliabilism, justification of a belief amounts to being formed by a process that is reliably truth-conducive, and knowledge reduces to reliably produced true belief. An issue that has been raised against this theory is the generality problem, which is based on the observation that while a specific belief is always generated by a specific belief-forming process ("token" process), reliability only makes sense relative to repeatable processes (process "types"). Now since all tokens seem to belong to multiple types, each of which might differ in how reliable they are, it is unclear how it is determined which specific type a particular token belongs to and, consequently, how reliable the process is (see, e.g., Goldman 1979, 1986, 2017).

Andreas Stephens

andreas.stephens@fil.lu.se

1 Lund University, Lund, Sweden 
To make the problem vivid, Conee and Feldman (1998) present an iconic example in which a person in an everyday situation looks out the window, sees a maple tree, and thus forms the belief that there is a maple tree outside. Conee and Feldman highlight how reliabilism needs to identify the type of the process causing the belief in the face of the fact that each token can be seen as belonging to multiple types:

The token event sequence in our example of seeing the maple tree is an instance of the following types, among others: visually initiated belief-forming process, process of a retinal image of such-and-such specific characteristics leading to a belief that there is a maple tree nearby, process of relying on a leaf shape to form a tree-classifying judgment, perceptual process of classifying by species a tree located behind a solid obstruction, etc. The number of types is unlimited. They are as numerous as the properties had by the belief-forming process. Thus, process reliability theories confront the question of which type must be reliable for the resulting belief to be justified. It is clear that the answer to this question will significantly affect the implications of the theory. [...] So, which type has to be sufficiently reliable? (Conee and Feldman 1998, pp. 2-3)

As many authors have pointed out (Goldman 1986; Adler and Levin 2002; Comesaña 2006; Olsson 2016; Kampa 2018), the generality problem is not a problem just for process reliabilism but for many other epistemological theories facing similar issues. Thus, whether or not an epistemological theory is affected by the generality problem, or a similar difficulty, is unlikely to be an interesting concern when deciding among epistemological rivals. Even so, it is unsatisfactory that a fully convincing answer to the problem seems to be lacking.

We take as our starting point an observation made by Conee and Feldman themselves:

The notion of reliability applies straightforwardly only to enduring mechanisms, such as an eye or a whole visual system, and to repeatable types of processes, such as the type: visually initiated belief formation. (Conee and Feldman 1998, p. 2)

It is, we will argue, indeed such a biologically centered perspective that stands the best chance of providing fruitful input to investigations of the generality problem.

Specifically, we will address two central concerns raised by Goldman and Beddor (2016; see also Olsson 2016; Goldman 2016):

Which repeatable type should be selected for purposes of assigning a reliability number to the process token? If no (unique) type can be selected, what establishes the justificational status of the resulting belief? (Goldman and Beddor 2016, section 3)

In response, we will investigate the hypothesis:

A token belief-forming process instantiates a uniquely "right type" of the biological agent's cognitive architecture (including other relevant embodied features) 
and background experience, consisting in the process of attractor-guided neural activation. ${ }^{1}$

In section 2, we present our approach. In section 3, we discuss how dynamical features and complexity are important to take into account in order to reach a biologically plausible interpretation of belief-formation, justification, and knowledge. Section 4 contains a detailed example of our approach in action. In section 5, we present considerations that can be used to identify the type that a token instantiates in a particular case. In section 6 , we mention and respond to a number of objections. In section 7 , finally, we summarize our results.

\section{Approaches to the Generality Problem}

Conee and Feldman (1998, pp. 3-5) identify three conditions that an answer to the generality problem should satisfy. A reasonable account should be principled, plausible, and true to the spirit of reliabilism. The first condition rules out ad hoc solutions lacking a proper foundation. The second condition rules out type assignments that make implausible correlations between reliability and justification. The third condition excludes solutions that are not in line with the reliabilist epistemological tradition. Furthermore, according to Conee and Feldman (1998, p. 5), "[i]t is reasonable to look for a solution to the generality problem in three places: common sense, science, and context." According to the common sense approach, the relevant types are those that we use in our daily life: "seeing," "hearing" etc. The scientific approach consults a relevant science for guidance as to how to fix the types of given process tokens. The contextual approach holds that the relevant types are relative to context.

Concerning the common sense-approach, Conee and Feldman (1998, p. 7) claim that "there are far too many common sense types to provide a unique identification of the relevant type for each process token [and] not all beliefs resulting from any one such type are even approximately equally justified." However, Jönsson (2013) and Olsson (2016) have convincingly undermined these claims by providing strong arguments showing that people do converge (in line with basic level effects) on the same type description when they report their classification of belief-forming processes and justifiedness - contrary to Conee and Feldman's claims (see, e.g., Rosch 1973; Webb and Graziano 2015; but see Jönsson 2015).

The approach we will heed instead focuses on scientific types. In particular, we will focus on scientific explanations based on cognitive science of how particular process tokens instantiate certain process types (see, e.g., Gigerenzer 1991; Goldstein and Gigerenzer 2002; Lee 2007; Rysiew 2017). Belief-formation processes take place in the natural world, and empirically guided scientific theories provide our best understanding of natural phenomena. Moreover, the part of the natural world in which beliefformation takes place is the brain of a cognitive agent. Relying on the best scientific accounts currently on offer is very much in the spirit of naturalized epistemology (Quine 1969), which is the framework within which most reliabilists arguably situate

\footnotetext{
${ }^{1}$ Both process tokens and process types should be understood as involving mostly automatic and nonconscious processes, an interpretation that will be motivated in the following sections.
} 
their theory. Importantly, we consider scientific types to involve contextual features, and we think that they can be used to understand common sense types. We hence consider it possible to unify the approaches highlighted by Conee and Feldman. We will return to this topic below where we will discuss how contextual factors influence how process tokens actually instantiates process types.

We are not the first to suggest a cognitively informed perspective on the generality problem: Alston $(1995)^{2}$ is another case in point. While we agree with Alston's observation that process tokens belong to specific natural kinds, we part company with him in that we do not consider conscious psychological processes to be the kind of processes we ought to focus on (see also Goldman 1979, 1986; Heller 1995):

If the epistemic status of a belief is a function of the reliability of the process that generates the belief, it is the reliability of the psychological process that is crucial. Looking at perceptual belief formation, no matter how exemplary and no matter how finely tuned the neural transformations involved in the pathway from the eye to the brain, if the belief is not formed on the basis of the conscious presentation (and/or its neural correlate) in a truth-conducive way, the belief will lack the epistemic desideratum that is stressed by reliabilism. (Alston 1995, p. 12, italics in original)

Since reliabilism is an externalist theory of justification and knowledge, Alston's demand for internalist transparency seems to be misplaced (cf. Comesaña 2006, pp. 30-31). The important aspect for the externalist account is, rather, the truthconnection - whether a belief is formed in a way that reliably connects it to the world (Goldman and Beddor 2016).

As we will try to make plausible, particular process tokens automatically and nonconsciously instantiate particular process types. It follows that an agent might not from her subjective first-person perspective be able to identify the relevant type in a given case. Moreover, the cognitive underpinnings of the process type will involve much more complexity than tends to be acknowledged even by authors with a cognitive bent. For these reasons, approaches that take the transparency of an agent's justification and knowledge for granted, demanding a linguistic rationalization concerning the correct type, are, in our view, biologically implausible.

Another naturalistic perspective on the generality problem is offered by Beebe (2004). He presents a two-step solution where he in the first step argues that a specific set of conditions must be satisfied by the relevant type. "According to the tri-level condition, cognitive process types are information-processing types that are partially defined by their computational and algorithmic properties" (Beebe 2004, p. 180). Here, Beebe follows Marr (1982) who presents a three-level hypothesis of cognitive processing (computational, algorithmic, and implementational). We consider this approach interesting but part way with Beebe (2004, p. 183) when he explicitly argues against the relevance of the implementational level claiming that "[a]lthough physical properties make important contributions to scientific explanations, they cannot help in selecting relevant cognitive process types." We consider this move to be problematic and will

\footnotetext{
${ }^{2}$ Alston focuses on what he calls psychological functions, habits, dispositions, or mechanisms. For a defense, see Adler and Levin (2002). See Feldman and Conee (2002) and Comesaña (2006) for criticism.
} 
below argue that the implementational level does offer fruitful input to the generality problem. In fact, as Beebe points out, whereas the computational and algorithmic levels are abstract, the implementational level directly addresses natural physical phenomena in the world. But, far from being problematic or irrelevant, we will show below that these physiological underpinnings of belief-formation do elucidate pertinent information concerning how token belief-forming processes can be assigned a uniquely right type. The tri-level condition step is, however, only seen as providing a first delimitation of relevant types and we will not here address the second step of Beebe's solution concerning statistical relevance.

We will now proceed to flesh out how process tokens instantiate process types, using an approach that centers on scientific explanations of the natural phenomena involved in belief-formations at an implementational level.

\section{A Dynamical Perspective on Process Types}

Cognitively speaking, a specific belief is always generated by a specific belief-forming process (a token), consisting in the activation of a particular neural pattern. A particular process token (of neural pattern activation) might intuitively seem to be assignable to different process types of varying reliability, suggesting that a cognitive or dynamical approach to the generality problem is a non-starter. However, we nowadays have significant theoretical insight and much relevant empirical evidence concerning human neural activity showing that belief-forming processes follow specific paths - attractors (which we will present and discuss below) (Buzsáki 2006; Lakoff and Johnson 1999; Kinzler and Spelke 2007).

Importantly, while there is a language-focused sense in which tokens hypothetically can be said to belong to multiple types, there is, in fact, an "explicit rule," to use Goldman's (2016) expression, operating in the cognitive domain: a given beliefforming token process instantiates a unique process type of attractor-guided neural activation. In this section, we will support this claim by shedding further light on the cognitive reality of belief-formation.

Since the occurrence of attractor-guided neural activation is ultimately an empirical question, we will now discuss how beliefs actually get formed, which is best elucidated by a pluralistic approach (Dale et al., 2009). As an organism interacts with its environment, statistical regularities will be learned and associated with suitable actions. This process can continue throughout an organism's lifetime, but the rate of learning tends to decrease with time. When stimuli, such as a physical object, are encountered by the organism, it will activate different pathways with an intensity depending on how well the stimuli fits the ideal or prototypical pattern associated with the different pathways. Although several pathways may be activated at the lower levels of perception, winner-takes-all mechanisms are in place to increase the probability that only a single process is engaged at the highest level. Furthermore, expectations from experience tend also to favor a single outcome by biasing some pathways over others (see, e.g., Ward 2002; Buzsáki 2006).

In order to address the generality problem, we will use a dynamical perspective focusing on dynamical systems, or systems that change with time, such as the brain. When constructing a dynamical model, one of the first tasks is selecting a set of state 
variables that describe how the state of the system progresses in time. Each state variable is a dimension in the system's state space. In a model of the nervous system, the state variables might include the activity of various brain regions, while in a model of a biological cell, the variables might be the concentration of various molecules. How the system as a whole evolves through time is called its trajectory, and is dependent on how the system is parameterized. In contrast to variables, parameters are static for a given trajectory. The complete set of trajectories corresponding to all possible settings of the system's parameters is called the flow of the system. By observing the flow of a system, it is possible to identify patterns in the trajectories. Such patterns are usually regions or points in the state space where trajectories tend to end up. Since these regions appear to be attractive to nearby trajectories, they are often called attractors. So, processes do not follow random paths, but rather automatically move towards specific ones. Buzsáki (2006) illustrates a (limit cycle) attractor as follows:

[T] hink of a racing car on a circular track. [...] The exact path of the car will vary somewhat in each round, bypassing other cars at different parts of the track, but this path variability is limited by the physical barriers of the track. The car can occupy any part of the track but should avoid the barriers. Thus, the track surface can be conceived of as the attractor of the car's orbit. (Buzsáki 2006, p. 137)

Using this metaphor, the car can be seen as a process token, whereas the track is the relevant process type. Every time the car (token) completes a lap, its tires affect the track (type), making the impressions deeper (the type more entrenched).

From a dynamical perspective, a discussion of the maple tree example will center on the human perceptual system, where primitive perceptual categories such as lines with varying orientation, and the complexes (such as branches, and later on whole maple trees) formed by these primitives further along the visual pathway, are attractors (scientific types) in the visual system's state space. A specific perceptual experience will then consist in a particular neural activation pattern that is the strongest, in accordance with the process of attractor-guided neural activation.

Now, we have argued that "the right" process type is to be identified with the process of attractor-guided neural activation, which clearly exemplifies Conee and Feldman's scientific types. However, an agent is affected by her context, and background experience, which is governed by, for example, evolutionary, developmental, social, and cultural factors. So, scientific types have clear elements of contextdependence built into them, in the sense that an agent's interplay with the external world affects which attractor is activated. Since most humans share a similar environment, the type that is instantiated (the process of attractor-guided neural activation) will — with small fluctuations depending on level of expertise - be the same for all agents. In section 5 below, we will reconnect to this topic at greater length. Given this, it is hardly surprising that agents report similar type assignments when prompted. Thus, it is in virtue of actual type-convergences (scientific types: attractors) concerning biological cognitive agents' mental and behavioral processes, including beliefforming processes, that our intuitive classifications (common sense types) tend to converge (Olsson 2016; Jönsson 2013). We thus believe that Conee and Feldman's 
three perspectives - common sense, science, and context - far from being mutually exclusive can be naturally combined in one unified account.

So, similar to how Beebe (2004) uses his first-step tri-level condition (on the computational and algorithmic levels) to demarcate relevant from irrelevant types, we have argued that the implementational level shows how there is a natural phenomenon underlying specific belief-formations amounting to a particular type - in Alston's parlance, a natural kind.

\section{A Concrete Example}

We here present a slightly more detailed account of the features involved in visual processing (seeing a maple tree), which is the scene that Conee and Feldman introduced. As we have claimed, a given belief-forming token process instantiates a unique process type of attractor-guided neural activation.

From two-dimensional information, agents are able to create a three-dimensional world. In short, segregating processes keep different objects separate, by means of, for example, visual processing of stimuli into foreground (object) and background. This is done by an interplay of bottom-up aspects and top-down aspects together forming a percept. Bottom-up aspects involve, for example, recognition of edges and other basic visual cues, whereas top-down aspects involve, for example, past learning and background experience. Grouping processes, on the other hand, let the agent assemble elements into wholes. Groupings thus enable Gestalts (meaningful wholes) to be perceived through factors such as proximity, similarity, common fate, continuation, and closure. An additional important factor that greatly affects what is perceived is the agent's attention, since the spotlight of attention governs what stimuli will be processed. Furthermore, the complete visual scene that makes up the agent's visual stimuli will, with its different cues, interact to form a contextual understanding in the agent (Kandel et al. 2013, pp. 611-615).

Another way to describe this whole process type is that low-level processings of orientation, color, contrast, disparity, and movement direction are translated into intermediate-level processings of color integration, surface properties, shape discrimination, surface depth, surface segmentation, and object motion, which then are integrated into a high-level identification, by way of tying together visual primitives with categorical and associative linkings, additional sensory signals, memories, emotional valence, and top-down predictions (Kandel et al. 2013, pp. 560, 622; see also Friston 2010; Clark 2013, 2015). Iterations of the visual perceptual process in general, as well as specific processes for particular stimuli, affect (form) the agent's cognitive faculties:

$[\mathrm{C}]$ ognition is nothing more (and nothing less) than a special kind of pattern formation, the interplay of functional segregation and integration and the continual emergence of dynamical structures that are molded by connectivity and subtly modified by external input and internal state. The shape of cognition, the nature of the information that can be brought together and transformed, is determined by the architecture of brain networks. The flow of cognition is a result of transient and multiscale neural dynamics, of sequences of dynamic events that unfold across time. The variety of cognition, the seemingly endless diversity of mental 
states and subjective experiences, reflects the diversity and differentiation made possible by the complexity of the brain. (Sporns 2011, p. 206)

In Conee and Feldman's described scene, the agent centers her attention on a specific place outside her window. A specific phenomenon (a maple tree) is segregated out as a foreground object, in contrast to the background, and a maple tree Gestalt is recognized. The encountered stimuli are thus processed both bottom-up and top-down, together governing what is perceived. As the example is presented, light reaches the agent's eye. The agent's retinal photoreceptors (cones and rods) register and convert the incoming stimuli and send the encountered information forward via retinal ganglion cells and the optic nerves towards other brain areas, in particular, to the lateral geniculate nucleus (LGN). From here, information can be sent to the amygdala, if the agent has strong emotional connotations to maple trees. If so, an emotional reaction, as well as automatic behavior, might ensue. Regardless of emotional content, information is forwarded to the primary visual cortex (V1-V3) of the occipital lobe at the back of the skull, where various specialized neurons process the information. Different cell layers are sensitive to different aspects such as the color of the maple tree and its leaves (parvocellular) and the movement - if there is any-of the maple tree's branches (magnocellular). The information is re-coded and then follows two central neural streams: the ventral stream and the dorsal stream. The ventral stream focuses on visual identification of what the stimuli is (components of a maple tree). This stream goes off towards the temporal lobe. The dorsal stream focuses on where the stimuli is located (outside the window, standing vertically) and how an agent might interact with the stimuli (pluck leaves, chop down). This stream moves towards the parietal lobe at the top of the agent's skull. At the early stages of these streams, only basic featurescan be detect. By combining these features it is nonetheless possible for the agent to combine, at later stages, this information into more complex representations, in for example regions such as V4 and V5/MT.

Moreover, top-down processes originating from prefrontal areas at the front of the agent's skull connect with the bottom-up processes. These processes enable the agent to predict its incoming stimuli (see, e.g., Friston 2010; Clark 2013, 2015) together with memories linked particularly to temporal lobe areas and the hippocampi (Mizumori 2013; Smith and Bulkin 2014). This aspect thus elucidates a particular feature of how the context governs perception. To clarify, if the context makes it salient that the object is to be interpreted as a maple tree, the agent can do so even before processing all the bottom-up stimuli. This is so since rather than elaborating on all individual stimuli, the agent can save energy by only tending to relevant prediction-errors (Friston 2010; Clark 2013, 2015; Hohwy 2013). This means that if the agent is well-acquainted with the scene outside her window she will not have to expend a lot of energy to categorize what she sees. Instead, her background experience makes her have certain preconceptions about the relevant scene. As long as there is nothing that the agent interprets as being out of the ordinary in the scene, she will quickly categorize what she sees (as a maple tree). ${ }^{3}$

\footnotetext{
${ }^{3}$ If the tree, for example, had been cut down, the agent would—most likely—have interpreted this gap as a prediction-error.
} 
What we intend to highlight with this outline is that the process type (the process of attractor-guided neural activation) involved in seeing a maple tree depends on very specific elements relevant for visual perception. Even though most brain functions involve a number of parallel processes, it is nevertheless the case that neural pathways for specific functions reside in specific brain areas. Indeed, these "[...] specific patterns of interconnection and the resulting functional organization of neural circuits in distinct brain regions underlie the individuation of behavior" (Kandel et al. 2013, p. 337). Hence, specific neural activations (tokens) do not take place in a black box. Instead, they follow specific pathways. And, even though all humans to a large extent overlap in how our cognitive architecture is shaped, all individuals have specific attractor pathways that are unique for them. Now, to reiterate, when someone sees a maple tree, a particular neural activation pattern will be the strongest, yielding a unique process of attractor-guided neural activation. This is the relevant type.

\section{The Role of Context and Social Factors}

Goldman and Beddor (2016, section 4) point out that it is generally assumed that "a 'solution' to [the generality] problem will consist in a formula for identifying a unique process type given any specified case and token (assuming the case is specified in reasonable detail)." As we have tried to make clear, this is a request that is possible to satisfy although doing so involves a high degree of complexity. In other words, the complexity of scientific types entails that, although there exists such a type in every concrete case, it might be difficult to describe or identify it. Furthermore, any particular specification can always be put in "theoretical doubt" by further demands concerning what amounts to reasonable detail. ${ }^{4}$

Above, we have identified how belief-forming process tokens instantiates a uniquely "right type" of the biological agent's cognitive architecture (including relevant embodied features) and background experience, consisting in the process of attractor-guided neural activation that can separate relevant types from irrelevant ones. Below, we elucidate a number of perspectives that delineates contextual and background experiential factors that influence how process tokens actually instantiates process types (Olsson 2016, pp. 180-181), where particularly hippocampal neuron ensembles play a crucial part regarding expectations and prediction-error detection of contextual features (see, e.g., Mizumori 2013; Smith and Bulkin 2014):

When a new context is encountered, a unique hippocampal ensemble is recruited to represent it. Memories for events that occur in the context become associated with the hippocampal representation. Revisiting the context causes the hippocampal context code to be re-expressed and the relevant memories are primed. As a result, retrieval of appropriate memories is enhanced and interference from memories belonging to other contexts is minimized. (Smith and Bulkin 2014, p. 52)

\footnotetext{
${ }^{4}$ Although, for example, Peirce (1877) would argue against the relevance of this merely theoretical possibility.
} 
As mentioned by Conee and Feldman, context is an important aspect to take into account regarding belief-formations:

Although a solution must be principled, it need not state necessary and sufficient conditions for relevance that are either precise or always determinate. Claims to the effect that a belief is "epistemically justified" might be vague and they might be context-sensitive in various ways. A solution must be universal only in that it must specify the relevant type whenever there are definite facts about justification. (Conee and Feldman 1998, p. 4)

The situational context (including social factors) we find ourselves in is then what governs which combination of factors is made salient and deemed relevant, which in turn determines which process of attractor-guided neural activation is instantiated. While Conee and Feldman classify context as a perspective of its own, we instead see it as an aspect that enters into a scientific perspective (scientific types). For a given process token, the context helps determine a process type as the process of attractorguided neural activation in that context (see, e.g., Alston 2005; Wunderlich 2003). For instance, depending on what amount of detail is required, different process types might be relevant. Although contextual differences exist, there are many more factors that remain across contexts. For example, evolutionary, developmental, social, and cultural factors are to a large extent universally present.

Concerning the development of human categorization abilities, Murphy (2002, p. 328) argues that human children develop the ability to distinguish basic level categories at around 2.5 years. This is followed by more abstract, superordinate categories at 4 years. The last to develop are more particular categories, or subordinate categories, which become available at about 5 years of age (Murphy 2002). Superordinate categorization intuitively implies being able to remove information from percepts, i.e., being able to do abstraction. However, according to Markman et al. (1980), it may be that children rather regard superordinate categories as collections of things. When considering that removal of detail in practice requires inhibition, a late-arriving ability in the history of evolution, the grouping hypothesis appears likely. The observation that subordinate categories take longer to develop than superordinate ones may also make sense from a perspective of statistics. Exemplars of subordinate categories are by nature sparse, hence it takes time for the child to have experienced a sufficient number of exemplars to arrive at the finer details. More generally, children tend to make use of heterarchies more often than proper hierarchies, and they appear to have a preference for a single level of classification (Murphy 2002, p. 327). In any case, the preference for basic level categories, as noted above, remains throughout adulthood (Murphy 2002).

Our social upbringing shapes our adult selves, involving for example parental and peer influence, as well as our position in dominance hierarchies. Moreover, social stereotypes tend to be used in order to arrive at fast judgments. Social animals have evolved the ability to efficiently understand each other through the development of body language and visual displays, scents, or noise patterns based on a similar perception of the world. Our biology thus limits the possibilities of divergent understandings of the world by facilitating similar interpretations of the world through the overlap of our DNA that code for the development of our sensory and communicative 
organs, and the structure of our interpretative brains. Humans are, as social beings, genetically predisposed to copy the type of higher-order cognitive processes other beings use to understand the world. Finally, cultural aspects such as education, norms, rituals, and "ways of lives" govern what we find "normal" and what we strive to accomplish.

From a dynamical and systems perspective (Mobus and Kalton 2015), natural phenomena can be investigated on different levels and from different perspectives. But assuming a naturalistic position, such investigations are mutually supportive rather than adversary. Importantly, it is not the role of philosophers, qua philosophers, to decide whether any particular science is correct or not. Hence, in response to theoreticians who think that the generality problem for reliabilism cannot be solved, we have argued that actual process tokens instantiates certain process types (attractors). Furthermore, we have pointed out a number of factors (evolutionary, developmental, social, and cultural) that separate relevant from irrelevant types and determine the existence of a unique type in a way that is in principle accessible to an investigator even though gaining such access would require a lot of work. However, if a solution to the generality problem is required to provide a procedure by means of which the unique process type in a given case can be easily identified, we believe that no such solution can be found - not because there are no unique types but because the demand vastly mischaracterizes the complexity of the world and our brains.

\section{Objections}

Conee and Feldman's arguments against the scientific approach center on Alston's (1995) account of natural kinds. As a first issue, they believe that "[m]erely citing the fact that each belief-forming process falls into a natural kind does not provide an adequate rule of relevance" (Conee and Feldman 1998, p. 10). They instead argue that "there is no good reason to think that each token belongs to just a single natural kind" (Conee and Feldman 1998, p. 10). As has been shown above, this is a mischaracterization of natural dynamical processes such as belief-forming processes. Process tokens do, in fact, belong to specific natural process types (attractors).

Since different sciences use different (terminological) categorizations, it is tempting to view them as indicating different natural kinds. Conee and Feldman use this point as basis for a second critique, although this also amounts to a mischaracterization. A certain natural phenomenon can indeed be described (investigated, modeled) by various sciences on different levels of abstraction. But this does not indicate that the natural phenomenon belongs to different natural kinds, rather a specific natural kind can be "triangulated" from many points of view (Horst 2016). Accordingly, a certain process of attractor-guided neural activation is an instantiation of a specific natural kind - although it can be described using different sciences.

Conee and Feldman continue by arguing against Alston's idea that a specific function of belief-forming process activation governs the relevant type. Above, we have mentioned, and argued against, Alston's claim that only internally salient types are relevant. But when this restriction is removed, Alston's account is basically correct-although it is not presented in a detailed manner. Conee and Feldman (1998, p. 11) mention how Alston's position entails that "there is only one actually 
operative 'psychologically real' type for each belief-forming process." and then critique Alston for being unclear regarding how the agent could "pick out" the correct type, out of all theoretically possible. We have argued that this is not something that the agent does consciously; rather, this is automatically done by non-conscious reflexive processes governed by the world.

Moreover, Conee and Feldman critique Alston for promoting functions that are maximally specific. It is then "only one function [which] is 'operative' in the formation of any belief" (Conee and Feldman 1998, p. 13). But this, according to Conee and Feldman (1998, p. 15), leaves reliabilist theories "unable to distinguish the epistemic status of lucky guesses that happen to be based on distinctive features from expert judgments based on well-understood classifications." As has been previously discussed, it is the world - not the agent - that is of importance regarding the governance of which type is relevant. This objection is therefore based on a faulty understanding of how tokens instantiates types.

Lastly, Conee and Feldman point out how "[t]here is no good reason to think that the types that are of greatest value for psychological explanation are uniformly helpful to reliabilist theories of justification" (Conee and Feldman 1998, p. 17; see also Baergen 1995). ${ }^{5}$ However, in the preceding sections, we have delivered precisely such an account.

\section{Concluding Remarks}

We have discussed how agents' process tokens, instantiating particular types, do, in fact, to a large extent, converge, albeit that the processes involved are complex. We have further argued that a unified interpretation of the generality problem is plausible, where scientific types are seen to incorporate contextual and common sense features. In order to identify the process type in a given case, the best we can do may be to inquire into the contextual, evolutionary, developmental, social, and cultural factors relevant to the agent's background experience.

Our discussion of the generality problem has been principled, epistemically plausible, and true to the spirit of reliabilism. Token belief-forming processes instantiate a uniquely "right type" of the biological agent's cognitive architecture (including relevant embodied features) and background experience, consisting in processes of attractorguided neural activation.

Acknowledgments We want to thank Martin L. Jönsson for numerous suggestions, and for him being exceedingly charitable with his knowledge and time. Thanks to James Beebe and our anonymous reviewers for comments.

Funding Open access funding provided by Lund University. The authors gratefully acknowledge support from Makarna Ingeniör Lars Henrik Fornanders fond, grant numbers FO2019-0005; FO2020-0004 and Stiftelsen Elisabeth Rausings minnesfond: forskning, grant number RFh2019-0226.

\footnotetext{
${ }^{5}$ Baergen (1995) argues that categorizations take place at lower levels, which then become more fine-grained on higher levels, and that this ability is what makes us able to identify relevant types.
} 


\section{Compliance with Ethical Standards}

Conflict of Interest The authors declare that they have no conflict of interest.

Open Access This article is licensed under a Creative Commons Attribution 4.0 International License, which permits use, sharing, adaptation, distribution and reproduction in any medium or format, as long as you give appropriate credit to the original author(s) and the source, provide a link to the Creative Commons licence, and indicate if changes were made. The images or other third party material in this article are included in the article's Creative Commons licence, unless indicated otherwise in a credit line to the material. If material is not included in the article's Creative Commons licence and your intended use is not permitted by statutory regulation or exceeds the permitted use, you will need to obtain permission directly from the copyright holder. To view a copy of this licence, visit http://creativecommons.org/licenses/by/4.0/.

\section{References}

Adler, J., \& Levin, M. (2002). Is the generality problem too general? Philosophy and Phenomenological Research, 65(1), 87-97.

Alston, W. P. (1995). How to think about reliability. Philosophical Topics, 23(1), 1-29.

Alston, W. P. (2005). Beyond "justification": Dimensions of epistemic evaluation. Ithaca: Cornell University Press.

Baergen, R. (1995). Contemporary epistemology. Fort Worth: Harcourt Brace College Publishers.

Beebe, J. R. (2004). The generality problem, statistical relevance and the tri-level hypothesis. Noûs, 38(1), $177-195$

Buzsáki, G. (2006). Rhythms of the brain. Oxford: Oxford University Press.

Clark, A. (2013). Whatever next? Predictive brains, situated agents, and the future of cognitive science. Behavioral and Brain Sciences, 36(3), 181-204.

Clark, A. (2015). Surfing uncertainty: prediction, action, and the embodied mind. Oxford: Oxford University Press.

Comesaña, J. (2006). A well-founded solution to the generality problem. Philosophical Studies, 129(1), 2747.

Conee, E., \& Feldman, R. (1998). The generality problem for reliabilism. Philosophical Studies, 89(1), 1-29.

Dale, R., Dietrich, E., \& Chemero, A. (2009). Explanatory pluralism in cognitive science. Cognitive Science, 33(5), 739-742.

Feldman, R., \& Conee, E. (2002). Typing problems. Philosophy and Phenomenological Research, 65(1), 98105.

Friston, K. (2010). The free-energy principle: a unified brain theory? Nature Reviews Neuroscience, 11(2), $127-138$.

Gigerenzer, G. (1991). How to make cognitive illusions disappear: beyond "heuristics and biases.". European Review of Social Psychology, 2, 83-115.

Goldman, A. I. (1979). What is justified belief? In G. S. Pappas (Ed.), Justification and knowledge (pp. 1-23). Dordrecht: Reidel.

Goldman, A. I. (1986). Epistemology and cognition. Cambridge: Harvard University Press.

Goldman, A. I. (2016). Reply to Olsson. In B. P. McLaughlin, and H. Kornblith (eds.), Goldman and his critics (pp. 197-199). (Philosophers and their critics; Vol. 16). Chichester: Wiley-Blackwell.

Goldman, A. I. (2017). What can psychology do for epistemology?: revisiting epistemology and cognition. Philosophical Topics, 45(1), 17-32.

Goldman, A., and Beddor, B. (2016). Reliabilist epistemology. In E. N. Zalta (ed.), The Stanford Encyclopedia of Philosophy (Winter 2016 Edition), URL $=<$ https://plato.stanford.edu/archives/ win2016/entries/reliabilism/>.

Goldstein, D. G., \& Gigerenzer, G. (2002). Models of ecological rationality: the recognition heuristic. Psychological Review, 109(1), 75-90.

Heller, M. (1995). The simple solution to the problem of generality. Noûs, 29(4), 501-515.

Hohwy, J. (2013). The predictive mind. Oxford: Oxford University Press.

Horst, S. (2016). Cognitive pluralism. Cambridge: MIT Press. 
Jönsson, M. L. (2013). A reliabilism built on cognitive convergence: an empirically grounded solution to the generality problem. Episteme, 10(3), 241-268.

Jönsson, M. L. (2015). Linguistic convergence in verbs for belief-forming processes. Philosophical Psychology, 28(1), 114-138.

Kampa, S. (2018). A new statistical solution to the generality problem. Episteme, 15(2), 228-244.

Kandel, E. R., Schwartz, J. H., Jessell, T. M., Siegelbaum, S. A., \& Hudspeth, A. J. (Eds.). (2013). Principles of neural science (5th ed.). New York: McGraw-Hill, Health Professions Division.

Kinzler, K. D., \& Spelke, E. S. (2007). Core systems in human cognition. Progress in Brain Research, 164, 257-264.

Lakoff, G., \& Johnson, M. (1999). Philosophy in the flesh: the embodied mind and its challenge to western thought. New York: Basic Books.

Lee, C. J. (2007). The representation of judgment heuristics and the generality problem. Proceedings of the Annual Meeting of the Cognitive Science Society, 29(29), 1211-1216.

Markman, E. M., Horton, M. S., \& McLanahan, A. G. (1980). Classes and collections: principles of organization in the learning of hierarchical relations. Cognition, 8(3), 227-241.

Marr, D. (1982). Vision. San Francisco: W. H. Freeman.

Mizumori, S. J. (2013). Context prediction analysis and episodic memory. Frontiers in Behavioral Neuroscience, 7, 132.

Mobus, G. E., \& Kalton, M. C. (2015). Principles of systems science. New York: Springer.

Murphy, G. L. (2002). The big book of concepts. Cambridge: A Bradford Book, MIT Press.

Olsson, E. J. (2016). A naturalistic approach to the generality problem. In B. P. McLaughlin, and H. Kornblith (eds.), Goldman and his critics (pp. 178-199). (Philosophers and their critics; Vol. 16). Chichester: WileyBlackwell.

Peirce, C. S. (1877). Illustrations of the logic of science: the fixation of belief. Popular Science Monthly, 12(November), 1-15.

Quine, W. V. O. (1969). Epistemology naturalized. In Ontological relativity and other essays (pp. 69-90). New York: Columbia University Press.

Rosch, E. H. (1973). Natural categories. Cognitive Psychology, 4(3), 328-350.

Rysiew, P. (2017). Naturalism in epistemology. In E. N. Zalta (ed.), The Stanford Encyclopedia of Philosophy (Spring 2017 Edition), forthcoming. URL = <https://plato.stanford.edu/archives/spr2017/entries/ epistemology-naturalized/>.

Smith, D. M., \& Bulkin, D. A. (2014). The form and function of hippocampal context representations. Neuroscience \& Biobehavioral Reviews, 40, 52-61.

Sporns, O. (2011). Networks of the brain. Cambridge: The MIT Press.

Ward, L. M. (2002). Dynamical cognitive science. Cambridge: The MIT Press.

Webb, T. W., \& Graziano, M. S. (2015). The attention schema theory: a mechanistic account of subjective awareness. Frontiers in Psychology, 6, 500.

Wunderlich, M. E. (2003). Vector reliability: a new approach to epistemic justification. Synthese, 136(2), 237262.

Publisher's Note Springer Nature remains neutral with regard to jurisdictional claims in published maps and institutional affiliations. 